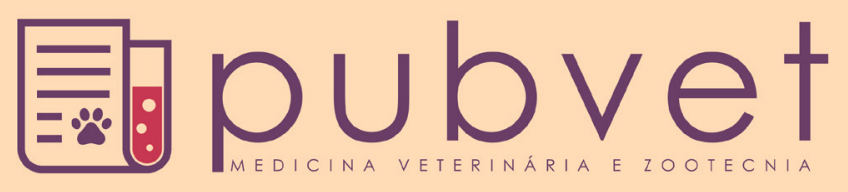

HTTP://DX.DOI.ORG/10.22256/PUBVET.V11N8.754-760

\title{
Zooantropologia: $O$ novo conceito dentro do velho em bem-estar animal
}

\author{
Matheus Hernandes Leira ${ }^{1 *}$, Jaqueline Roberta Cardoso Santos ${ }^{2}$, Lucas Silva Reghim ${ }^{3}$, \\ Lívia da Silva Ciacci ${ }^{1}$, Bianca Batista Barreto ${ }^{1}$, Luciane Tavares Cunha ${ }^{1}$, Daniela Ribeiro \\ Cazelato Amorim², Bruna Colares Alegro Belato², Marlon Ribeiro Bueno ${ }^{2}$, Debora Alves \\ Silva $^{2}$, Natalia Pereira Dias ${ }^{2}$ \\ ${ }^{1}$ Professor e Pesquisador do Curso de Medicina Veterinária, Centro Universitário do Sul de Minas - UNIS, Varginha-MG - Brasil. E-mail. \\ matheus.hernandes@unis.edu.br.*Autor para correspondência \\ ${ }^{2}$ Discente do Curso de Medicina Veterinária, Centro Universitário do sul de Minas - UNIS, Varginha-MG-Brasil. \\ ${ }^{3}$ Discente do Curso de Medicina Veterinária, Iniciação Cientifica, Centro Universitário do sul de Minas - UNIS, Varginha-MG-Brasil.
}

RESUMO. A relação homem e animal sofreu várias modificações ao longo de décadas, a princípio o homem era tido como o centro do universo, uma visão antropocêntrica, que fazia com que os animais fossem colocados em posição de inferioridade e subjugados pelos seres humanos. Partindo-se deste princípio, os animais eram tratados como seres sem sentimentos e indolores, tanto que eram submetidos a procedimentos de cunho científico sem qualquer tipo de zelo. Estes conceitos começaram a se alterar através de Darwin, que acreditava que o homem era a fruto da evolução animal e não uma criação divina. A partir de então, a conotação de homem como centro de todas as coisas começou a perder força. Posteriormente, surge então a zooantropologia que é um ramo da ciência que estuda as relações entre humanos e outras espécies com o objetivo de fornecer respostas a questões da interação homem e animal, visando obter contribuições à partir da análise da diversidade animal. Esta ciência admite um verdadeiro diálogo entre humanos e não-humanos e acredita que os seres humanos têm construído muito de seus predicados através da referência ao animal não-humano por intermédio de um processo de reciprocidade, em que a relação é em primeiro lugar "o que une os interlocutores", não sendo um fim em si.

Palavras chaves: Bem-estar animal, teoria da zooantropologia, senciência

\section{Zooanthropology: The new concept within the old on animal welfare}

\begin{abstract}
The man-animal relationship underwent several modifications over decades, at first man was regarded as the center of the universe, an anthropocentric view, which made the animals placed in an inferior position and subdued by humans. Starting from this principle, the animals were treated like beings without feeling and painless, so much that were submitted to procedures of scientific nature without any type of zeal. These concepts began to change through Darwin, who believed that man was the fruit of animal evolution and not a divine creation. From then on, the connotation of man as the center of all things began to lose strength. Subsequently, then comes the zooanthropology that is a branch of science that studies the relationships between humans and other species with the objective of providing answers to questions of human and animal interaction, seeking to obtain contributions from the analysis of animal diversity. This science admits a true dialogue between humans and nonhumans and believes that humans have built much of their predicates through reference to the nonhuman animal through a process of reciprocity, where the relation is first "the That unites the interlocutors ", not being an end in itself.
\end{abstract}

Keywords: Animal welfare, zoananthropology theory, sense 


\section{Zooantropología: El nuevo concepto dentro de lo antiguo en bienestar animal}

RESUMEN. La relación entre hombre y animal ha sido objeto de varios cambios a lo largo de décadas, al principio el hombre fue considerado como el centro del universo, una visión antropocéntrica, lo que hizo que los animales fueran colocados en posición inferior y subyugados por los seres humanos. Partiendo de este principio, los animales fueron tratados como seres sin sentimientos y sin dolor, por lo que fue sometido a procedimientos de carácter científico sin ningún celo. Estos conceptos empezaron a cambiar a través de Darwin, que creía que el hombre era el resultado de la evolución animal y no una creación divina. Desde entonces, la connotación del hombre como centro de todas las cosas comenzó a perder fuerza. Más tarde, surge la zooantropologia que es una rama de la ciencia que estudia las relaciones entre humanos y otras especies con el fin de dar respuestas a las cuestiones de la interacción hombre e animal, visando obtener contribuciones a partir del análisis de la diversidad animal. Esta ciencia permite un verdadero diálogo entre humanos y no humanos y cree que los seres humanos han construido gran parte de sus predicados por referencia a un animal no humano a través de un proceso recíproco en el que la relación esta en primer lugar "lo que une a los interlocutores", no siendo un fin en sí.

Palabras clave: Bienestar de los animales, teoría zooantropologia, sinciencia

\section{Introdução}

A zooantropologia está enraizada no ambiente teórico e cultural que desde os anos 70, tentou dar respostas para o benefício animal e as contribuições surgiram a partir da relação com outras espécies, em particular na educação e bemestar de terapia animal de estimação.

Existem quatro principais alvos teóricos de sua crítica: a psicologia, que vê o animal não humano, como um estímulo ou como um substituto para uma ligação emocional; a antropologia, que leva o conceito de "bom animal para pensar", ou o animal como um objeto útil para os processos de simbolização e categorização real; etologia escopo, que considera a propensão humana de interagir e avançar para as outras espécies.

A partir dos anos 90 em diante, a zooantropologia adquire identidade própria, graças à pesquisa na França de Hubert Montagner (Montagner and Alaméda, 1995) sobre o valor educacional da reunião entre a criança e o animal e as pesquisas de Roberto Marchesini, Sabrina Tonutti, Eleonora Fiorani, entre outros autores. Hibridativo o significado de a relação entre o ser humano e o eterospecifico na construção de predicados humanos (Montagner and Alaméda, 1995). Durante as duas últimas décadas do século XX, dentro dos chamados Estudos de Animais, há duas disciplinas mais focadas de caráter descritivo e explicativo, principalmente em relação à conotação prescritiva da bioética animal e a relação homem $\mathrm{x}$ animal.
A antrozoologia, Anglo-saxônica, com um tipo de corte transdisciplinar; e a zooantropologia, que cresce principalmente na Itália e na França, com a maior epistemológica ou teoria do conhecimento que foca na comparação com o caráter peculiar da relação com os animais.

\section{Zooantropologia}

A zooantropologia se desenvolve como disciplina específica há aproximadamente 20 anos na Europa e Estados Unidos, com o objetivo de fornecer respostas às questões da interação homem e animal. A zooantropologia se utilizou de pesquisas desenvolvidas no campo da bioética animal e a na afirmação da tese continuísta, que considera as diferenças entre seres humanos e não humanos como diferenças de grau, não de natureza, estendendo a noção de pessoa também aos animais. O mesmo autor entende que a zooantropologia e a bioética animal são verdadeiras revoluções de ordem filosófica, ética e pedagógica (Tugnoli, 2006).

Fonseca (2009) destaca Peter Singer que foi uma grande influência no movimento de libertação animal, em sua obra Libertação Animal ele argumenta contra o especismo, que é a discriminação contra certos seres por pertencerem a determinadas espécies. Seus questionamentos relacionavam-se em colocar chimpanzés em gaiolas de laboratório e contagiá-los intencionalmente com doenças humanas fatais, e o porquê de não fazer o mesmo com humanos que apresentam nível intelectual deficitário; que para 
ele é a consolidação do paradigma mecanicistautilitarista na relação homem-animal. Todavia, nem sempre foi assim. Essa ideia de consideração ao sofrimento animal, o filósofo René Descartes foi quem lançou o paradigma mecanicistautilitarista da relação homem e animal. Segundo Fonseca (2009), ele acreditava que os animais não possuíam espírito, bem como qualquer racionalidade ou sensibilidade e, de acordo com sua teoria, o animal ignora o sofrimento. $\mathrm{O}$ gemido, o uivo ou o grito dos animais não humanos passava a estar associado à disposição dos seus órgãos, representando meros reflexos externos.

Partindo do pensamento de Descartes, as práticas de vivissecção tornaram-se procedimento trivial no âmbito científico. Os animais, amarrados pelos membros, eram abertos, sem anestésicos (pois estes só foram descobertos em 1846), muitas vezes sob os olhares de civis, que pagavam para observar tais tipos de experimentação. Todavia, já nessa época, delimitavam-se os contornos de um problema moral envolvendo a exploração animal.

O grande ponto de virada nesta história surge com o pensamento darwiniano e a sua teoria da evolução. Os autores Silveira and Custódio (2011) deixam isso claro ao salientarem que Darwin já havia escrito que o homem, fazendo uso de sua arrogância, acredita ser uma grande obra, merecedora de uma intervenção divina, quando Darwin acredita que na verdade o homem é criado a partir de um animal. Darwin foi cauteloso em suas ponderações, uma vez que esse seu ponto de vista foi tecido antes de sua obra a Origem das Espécies em 1859; com isso seu pensamento acabou colocando em xeque qualquer forma de antropocentrismo, ou seja, do homem como sendo fruto de uma criação divina apartado de toda relação de ascendência com os animais. Essas mudanças de compreensão da relação homem e animal passam a ter, então, o potencial de retirarnos da posição central do universo. Depois de Darwin, a questão animal, que era considerada um tabu no universo acadêmico, passa a ser explorada. O pensamento darwiniano vai se edificando com a descoberta de habilidades matemáticas, a revelação da existência de cultura e a confecção e utilização de ferramentas por primatas e chimpanzés, além de uma enorme variedade de habilidades cognitivas complexas possuídas por esses animais.

Os anos 70 passam a ser considerados como do movimento de libertação animal, impulsionado pela reflexão de filósofos. Silveira and Custódio (2011) salientam que se passou então a questionar o status moral dos animais não humanos ao incorporar o argumento dos casos marginais. Neste momento o questionamento que se leva em consideração é o fato de que pessoas com retardo mental, bebês ou idosos, possuem algum status moral, uma vez que suas condições são bem próximas a dos animais. Desta constatação advém o seguinte questionamento: qual o motivo deste especismo em relação aos animais?

Marchesini (2005) acredita que o mito da pureza, a submissão dos animais ao homem e a ideia existencialista da forma perfeita foram as bases de toda a forma de discriminação. Ele aponta que Santo Agostinho já havia entendido isso, pois onde há discriminação humana versus não humano e maltrato aos outros viventes, há o modelo para submeter o homem ao homem. $\underline{\text { Sordi }}$ (2011) acrescenta a discussão o fato de que nas últimas três décadas novos paradigmas estão em desenvolvimento na antropologia como os conceitos de natureza confrontando com cultura, humanidade com animalidade, abrindo espaço à antropologia ecológica. Moran (1994) define antropologia ecológica como o estudo das populações dentro de um determinado ecossistema.

Outras áreas além das ciências humanas têm abordado a relação dos homens e os animais, como a biologia, a genética, e a primatologia, utilizando inclusive conceitos de cultura e política, próprios das ciências humanas para tratar do tema da sociabilidade animal.

Um movimento de defesa animal que surge ao mesmo tempo em que a antropologia das interações entre humanos e animais é o chamado abolicionismo ou libertarismo. $\mathrm{O}$ abolicionismo tem como objetivo combater qualquer tipo de utilização dos animais pelos humanos, diferentemente do movimento bem-estarista que seria um movimento popular de defesa dos animais. O bem-estarismo aceita o uso humano dos animais na medida em que eles sejam tratados humanitariamente, isto é, que se evite seu sofrimento desnecessário e se funda na regulamentação do tratamento animal. Já o movimento abolicionista acredita que todos os seres sencientes têm o direito de não serem usados exclusivamente como meios para os fins de outros.

Segundo Cohen and Regan (2001) esse movimento abolicionista é contra o uso de animais para quaisquer fins, até mesmo para o uso em 
pesquisa científica, para ele não bastam gaiolas maiores é necessário que elas não existam. Esse movimento se propõe a ser a voz dos animais, uma vez que eles não possuem uma, propriamente dita.

Apesar de ambos, o abolicionismo e o bemestarismo terem como principal preocupação os animais, o abolicionismo critica o bem-estarismo em alguns aspectos, como quando no acolhimento de cães e gatos, que pode ocasionar um especismo cruzado, o que para Sordi (2011) é definido como uma hierarquização dos animais, uns sobre os outros, uma espécie sobre a outra. Como, por exemplo, o cachorro e gato passaram a ser membros da família; enquanto outros são somente números em abatedouros.

Em termos de Brasil há poucas, mas interessantes pesquisas na área de sociabilidade animal, como a realizada por Guilherme José Silva de Sá (Sá, 2011) que observou primatas em suas interações sociais. Em sua tese de mestrado, Oliveira (2006) estudou a relação entre cães e seus tutores, abordando em diferentes perspectivas. Foi sancionada a Lei n. 11.794/08, chamada Lei Arouca que regulamenta a experimentação animal e estabelece parâmetros e procedimentos mais rígidos para isso. A referida Lei 11.794 estabelece que Comissões de Ética Institucionais ao Uso de Animais (CEUA) e tem como função regulamentar o uso de animais em Universidades e instituições de ensino que fazem pesquisa nesta área.

A antrozoologia utiliza pesquisas etológicas e antropológicas, com o objetivo por um lado, para descrever as características da relação homemanimal, e por outro para estudar suas aplicações potenciais. Os pressupostos que são apresentados para explicar esta necessidade interespecífica são diferentes. Existe a hipótese de "Biophilia", proposta por Edward (Wilson, 1984), como características inatas de nossa espécie que de alguma forma fascinados por formas de vida. Existe a hipótese conhecida como "decepção dos pais", proposta por James Serpell (Serpell, 1986), um engano trama durante a evolução por animais domésticos que estavam a tornarem-se parasitas do homem em termos de cuidados parentais.

De acordo com a hipótese de cultura há um interesse nos animais como sistema de abertura do homem, expressão realizada, por exemplo, por Digard (1990). Depois, há outra maneira de interpretar a relação homem-animal ou para considerá-lo, uma necessidade humana específica, um epifenômeno de características humanas.
A sub-rogação possui necessidades de outras especificidades em geral, podemos dizer que os estudos em animais têm uma dívida considerável para a pesquisa antropológica, mesmo a pesquisa etológica que definitivamente Konrad Lorenz um dos fundadores, tem-se interessado nas interações interespecíficas, com especial atenção para a relação homem-animal (Lorenz, 2011). Especificamente, a busca de impressão para Lorenz oferece a possibilidade de analisar as estruturas morfológicas e comportamentais capaz de saltar as barreiras entre espécies e estar ativo no transpecífico que é a maneira da especificidade que está para ser encontrada em um substrato comum de sinais que podem ser mais ou menos decodificada para além da barreira das espécies. Uma delas é a biossemiótica da juventude universal, um catálogo de características morfológicas, o arredondamento do crânio, a proeminência da frente, o maior volume dos olhos, o repertório lúdico que eles são capazes de evocar, ao longo da fronteira da espécie, atitudes de cuidado e carinho.

Seguindo o estudo etólogo da matriz de James Serpell, ganhou teoria atraente sobre o link que está a ser estabelecida entre o homem e o animal credenciado pelo processo de domesticação. A partir do fato indiscutivel da interespecífica adoção presente no homem e a tendência a diminuir a relação com o animal de estimação em um tronco parental, Serpell vê o processo de domesticação como uma espécie de parasitismo.

Uma visão diferente naturalista de Juliet Clutton-Brock para o qual o processo de domesticação é o resultado de uma combinação de fatores preparados no Paleolítico, quando o caçador "homem" e os "coletores" tinha visitas frequentes com o mundo animal e paralelamente, os animais tiveram uma drenagem inferior de distância, mas consagrado pela revolução neolítica com a posse total do território. De acordo com o papel de Clutton-Brock o homem na domesticação é absolutamente fundamental, embora capaz de abrir o caminho para a parceria (Lorenz, 2011).

Outros estudiosos como Paul Shepard, salientaram a importância da relação de caça para alimentação do próximo, o processo de domesticação. A comparação com o animal é principalmente jogada na derrota do tamanho, a competição por recursos, a necessidade de agarrar a carne.

Outra linha de interpretação em vez de a consideração de que o animal é para o homem algo 
especial, a prioridade máxima na avaliação da realidade externa. Um dos primeiros a considerar esta unidade incondicional para com os animais não humanos foi o entomologista Edward $\mathrm{O}$. Wilson, o pai da sociobiologia, para o qual você deve falar em seres humanos de uma "Biophilia" específica entendida como uma unidade motivacional para o mundo animal (Wilson, 1984).

\section{Diferenças interdisciplinares}

A diferença fundamental entre a zooantropologia e outras áreas de pesquisa sobre a relação homem e animal como a antrozoologia ou Estudos de Animais, é a recusa de uma abordagem multidisciplinar e a construção de sua própria estrutura epistemológica com base em alguns fundamentos.

A leitura animal não humano como alteridade ou como entidade na diversidade é, no entanto, equipado de subjetividade. $\mathrm{O}$ conceito de relação de diálogo entre o ser humano e animal não humano, admitindo uma multiplicidade de níveis de encontro com a alteridade animais e rejeitando assim plano causal, a ideia de que a relação com o animal não humano torna disponível para as contribuições da mudança para o ser humano ou a presença de umas "referências animais", a hipótese de que o encontro com o animal não humano pode assumir não só um aspecto fenomenal, mas pode ser uma epifania ou uma inspiração para os seres humanos (Marchesini, 2005). A admissão de que há predicados de origem da cultural humana devem ser considerados como fruto híbrido com o animal não-humano e não como um homem animal (Tonutti, 2012).

\section{Uma nova perspectiva}

A zooantropologia é muitas vezes difícil de compreender e aceitar porque vai contra alguns conceitos pré-estabelecidos. Aqui estão alguns: que os seres humanos não podem se comunicar, com eles mesmos, no sentido de intercâmbio de conteúdo com animais não humanos; que a cultura é o resultado criativo e o ser humano autossuficiente, o que seria em última análise autônomo e auto referencial na sua formação e realização de identidade; que a relação com o animal não humano é explicado pela identificação do causal coordenada ordem funcional instrumental, tais como "usado para", "animal", "é funcional para"; que o ponto de análise devem estar localizadas apenas nos dois termos da relação (animal humano e não humano) por suas características intrínsecas; que o animal não humano não tem um papel social na sociedade humana, mas que entra como um substituto para um outro ser humano ausente ou como fetiche compensatório de uma necessidade não satisfeita, em outras palavras, como uma aproximação ao homem.

\section{O papel de não humano}

Se considerarmos o fio comum que une a estas declarações você percebe imediatamente que é o vazio do significado relacional do não humano, em sua concepção autêntica e assim: subjetiva, original, dialógica, referencial na ideia antropocêntrica relação dialógica (único homem conversa com o seu companheiro ser humano) e forma autossuficiente de sua identidade (homem aprende apenas a partir de seu companheiro ser humano), e consequentemente de um animal não humano, papel negação social e relacional (Lestel, 2004).

São reservados para funções não humanos e tarefas realmente não relacionais (no sentido de que é dialógica transacional), mas a performativa, interativa ou compensatória, a zooantropologia vai contra estas afirmações, porque, ela admite um verdadeiro diálogo entre humanos e não humanos; ela acredita que os seres humanos têm construído muito de seus predicados pela referência ao animal não humano; ela argumenta que a relação é multidimensional, porque várias podem ser as causas implementarias e ativos assumidos pelo relatório; salienta que a multidimensionalidade exige que sugerem em análise não apenas os dois termos da relação, mas também o tipo de vínculo que os une que a configuração original do relatório; tem como pedra angular do pressuposto de que o animal não humano tem um papel, distintivo em virtude do seu ser não humano, na sociedade humana.

\section{A teoria da zootropia}

De acordo com o zooantropologo italiano Roberto Marchesini, que considera a zootropia como um evento de auto-implementativo e recursiva, onde não é possível considerar o ser humano como pura entidade que está relacionada, sem misturar com o animal não humano, mas, como uma entidade que em sua emergência como um ser humano já foi introduzido o eterospecifico, em que qualquer relação com o animal não humano altera o estado do ser humano ontológica. Em particular, a zooantropologia destaca dois eventos que transformam a maneira como 
pensamos a dialética interativa entre a outra espécie humana e o conceito de "relacionamento", que a reciprocidade de conteúdo e operação evolutiva, que deve ser investigado como uma entidade em seu configure ou características específicas em contratação. Especificamente, para a zooantropologia, a relação entre o homem e animal não humano não é a razão simples, neste caso, o foco de investigação é ainda sobre as duas figuras em interação, mas é entidade de emergência que é afetada não só de "identidade de dialoga, mas o tipo de relação" (motivações, atividades trocadas, papéis assumidos, expectativas) que os une em um determinado momento. Neste sentido, o relatório é uma superveniente entidade como um todo e que embora não negligencia a identidade de dialogar, de fato não considera essas variáveis como prevalente como, por exemplo, se um menino e uma menina são amigos sua configuração relacional é bastante diferente do que eles estão no amor, embora suas identidades permanecem os mesmos.

Para a zooantropologia a relação é em primeiro lugar "o que une os interlocutores", ou seja, que reciprocidade meio e transacional mesmo capaz, em alguns meios de transcender a identidade da relação pessoas. A zooantropologia está mostrada como "entidades dimensionais" ou uma a estrutura que se caracteriza antes de tudo com o tipo de meio de reciprocidades e estabelecimentos que realiza e exprime esse evento contextualizado. $\mathrm{O}$ conceito de "referência", entendida como contribuições evolutivas que o relatório faz algumas do dialogar com a disposição que especifica as coordenadas do desenvolvimento expressivo e ontogenético. Este conceito está no centro da pesquisa zooantropologica e, portanto, exige uma definição concisa para diferenciá-lo dos de uso performativa (o animal como produtor de desempenho), a construção de lote de material (o animal como uma paleta de cores para o artista homem), de entidade de substituição (a compensatório animal, vicária). Para zooantropologia a relação não é um fim em si, mas, na sua configuração tridimensional, produz uma referência que é caracterizada, em virtude da diferença de seu animal não humano, ou efeito de limiar e epifania ontológica da alteridade, em relação ao tamanho específico de relatório ativado na situação particular ou o efeito de endereço evolutivo.

Estas são duas transformações conceituais extremamente importantes que podem mudar o foco da investigação, entidades postas em causa, as metodologias de investigação, a aplicações coordenadas.

\section{Aplicações práticas}

A zooantropologia é algo complementar a etnobotânica, que estuda as relações entre as diferentes culturas e o uso de plantas (como alimentos, medicamentos, matérias-primas, etc. Também na literatura, rituais e vida social). $\mathrm{O}$ termo fitoantropologia é usado em vez de uma forma completamente casual.

Exemplos de áreas de estudo: laços emocionais (emoções) ou relação entre seres humanos e animais; percepções e crenças do homem em relação a outros animais (Sax, 2013), o impacto de alguns animais em comunidades humanas; mudanças em tais relações entre as culturas e ao longo do tempo, estudar o processo de domesticação e sua história (Tonutti, 2012).

\section{Conclusão}

A zooantropologia é uma ciência, oriunda dos Estados Unidos e Europa e que surgiu antagônica aos nossos antigos paradigmas, que começaram a ser desfeitos desde a publicação da Origem das Espécies, por Charles Darwin. Constitui uma área de estudo que recusa a multidisciplinaridade e forma sua própria epistemologia, tendo como objetivos estudar a interação entre seres humanos e não humanos, e os motivos que causam as relações entres esses seres em um determinado momento. A zooantropologia investiga a identidade da relação dos seres e não os seres em si, o que na contemporaneidade, visto o aumento de animais domesticados tanto para produção quanto de estimação, tornou-se uma ciência essencial para que haja um crescimento da harmonia no convívio entre humano e não humano.

\section{Referências Bibliográficas}

Cohen, C. \& Regan, T. 2001. The animal rights debate. Rowman \& Littlefield.

Digard, J.-P. 1990. L'homme et les animaux domestiques: anthropologie d'une passion. Fayard, Paris.

Fonseca, L. S. G. 2009. Hans Jonas e a responsabilidade do homem frente ao desafio biotecnológico. Universidade Federal de Minas Gerais, Belo Horizonte.

Lestel, D. 2004. L'animal singulier. Seuil, Paris. 
Lorenz, K. 2011. L'etologia: fondamenti e metodi. Bollati Boringhieri.

Marchesini, R. 2005. Fondamenti di zooantropologia. Perdisa, Bologna.

Montagner, H. \& Alaméda, A. 1995. L'enfant, l'animal et l'école. Bayard; AFIRAC, Paris.

Moran, E. F. 1994. Adaptabilidade Humana: uma Introdução à Antropologia Ecológica. Edusp.

Oliveira, S. B. C. 2006. Sobre homens e cães: um estudo antropológico sobre afetividade, consumo e distinção. Departamento de Veterinária. Universidade Federal do Rio de Janeiro, Rio de Janeiro.

Sá, G. J. S. 2011. " Meus macacos são vocês": Um antropólogo seguindo primatólogos em campo. Revista Anthropológicas, 16, 41-66.

Sax, B. 2013. Imaginary animals: The monstrous, the wondrous and the human. Reaktion Books, London.

Serpell, J. 1986. In the company ofanimals. Blackwell, New York.

Silveira, C. A. \& Custódio, A. E. I. 2011. O fazer o bem sem olhar a quem e os limites da abordagem antropocêntrica na história das relações homem-animal. ComCiência, 1-11.
Sordi, C. 2011. O animal como próximo: por uma antropologia dos movimentos de defesa dos direitos animais. Cadernos IHU ideias, 9, 3-28.

Tonutti, S. 2012. Zooantropologia. Gli animali nelle culture umane, Trattato di biodiritto. Giuffré, Roma.

Tugnoli, C. 2006. La Comunidad Economica Europea quiere que sus bosques funcionen. Revista do Instituto Humanitas, 200, 25-26.

Wilson, E. O. 1984. Sociobiology and biophilia: The human bond to other Species. Harvard University Press, Cambridge, Mass.

\section{Article History:}

Received 29 April 2017

Accepted 30 May 2017

Available on line 20 June 2017

License information: This is an open-access article distributed under the terms of the Creative Commons Attribution License 4.0, which permits unrestricted use, distribution, and reproduction in any medium, provided the original work is properly cited. 